\title{
THE EFFECT OF TEMPERATURE ON MEIOTIC TRANSMISSION RATES OF THE B CHROMOSOME OF MYRMELEOTETTIX MACULATUS (ORTHOPTERA: ACRIDIDAE)
}

\author{
M. W. SHAW* AND G. M. HEWITT \\ School of Biological Sciences, University of East Anglia, Norwich NR3 7TJ, U.K. \\ Received 19.xii.83
}

\section{SUMMARY}

Meiotic drive of B-chromosomes in Myrmeleotettix maculatus females decreased slightly during the course of egg-laying but was not detectably different under different temperature regimes. A proportion of males, however, showed substantially reduced transmission of the $B$ chromosome when maintained at lower temperatures. This could explain some of the geographic variation in B chromosome frequency that has been observed.

\section{INTRODUCTION}

The B chromosome polymorphism of the heathland grasshopper Myrmeleotettix maculatus is restricted to the southern and central parts of the U.K., although the animal itself is much more widespread (e.g., Hewitt and John, 1967). This restricted range may be due in part to historical and geographical accidents-there is evidence that in at least one part of the boundary of the B chromosome distribution the B may still be spreading (Shaw, 1983). However, it is also likely that the equilibrium B frequency varies from place to place. For example, Hewitt and Ruscoe (1971) examined both microclimatic variables and B frequency in a series of populations along a $1 \mathrm{~km}$ transect in the Welsh hills near Aberystwyth. It was clear that where the microclimate was cooler, the B frequency was lower. This suggests that the B might be restricted to the southern U.K. by climatic factors. Thus, an essential ingredient in a convincing explanation of the geographic distribution of B chromosomes in $M$. maculatus must be some understanding of how climate might influence equilibrium frequency.

The equilibrium frequency of B's in a population is probably set by the interaction of two forces. A net meiotic drive tends to increase the frequency in each generation, but is balanced by strong selection against 2B karyotypes, tending to reduce the frequency (Shaw, 1984). Hence differences in equilibrium $B$ frequencies between localities might be explained by differences in either meiotic drive or selection. In turn, such differences might be induced by features of the local environment or attributable to differences in the genetic makeup of the population in different places.

In this paper, we wish to describe an experiment designed to examine the effects of temperature on meiotic transmission rates of the $\mathrm{B}$ chromosome in $M$. maculatus. Temperature was chosen because it is the climatic variable that seems to be most closely associated with B frequency in studies of

\footnotetext{
* Present Address: Long Ashton Research Station, Long Ashton, Bristol BS18 9AF, U.K.
} 
distribution (e.g., Hewitt and Ruscoe, 1971, Hewitt and Brown, 1970, Robinson, 1973) and is convenient to control.

\section{Design and Methods}

Many orthopteran species seem to use radiant heat, when it is available, to regulate their internal temperature (e.g., Gregg, 1981). Certainly, in the absence of radiant heat, it is extremely difficult to rear $M$. maculatus in the laboratory (Kelly-Stebbings and Hewitt, 1972). Past experiments on this topic by one of us (GMH) have not been very successful for this reason. These experiments did, however, allow us to identify the broad range of tolerable experimental environments. As a first step, therefore, it seemed worth rearing nymphs through the vulnerable early stages in standard laboratory culture, and then looking for effects of the adult and late nymphal environments on meiotic transmission rates. This has the added advantage that, by changing environments during adult life, the effects of variation between grasshoppers can be examined independently of environmental influence on an individual grasshopper.

Three environments were used, chosen in the light of the experiments mentioned above. The standard laboratory conditions were $16 \mathrm{~h}$ with incandescent light providing a source of radiant heat, followed by $8 \mathrm{~h}$ of darkness. The background temperature was $27^{\circ} \mathrm{C}$ during the day, falling to $23^{\circ} \mathrm{C}$ at night. A hot ("continental") controlled environment was provided in a "Conviron" cabinet set to $14 \mathrm{~h}$ light at $36^{\circ} \mathrm{C}$ and $10 \mathrm{~h}$ darkness at $12^{\circ} \mathrm{C}$, while a cool ("maritime") environment was provided by another cabinet set to $14 \mathrm{~h}$ light at $32^{\circ} \mathrm{C}$ with $10 \mathrm{~h}$ darkness at $20^{\circ} \mathrm{C}$. These will be referred to as "control", "hot", and "cold" environments respectively.

Two sibships were used as sources of grasshoppers containing B's. Both were known in advance through karyotyping samples of eggs to contain a very high proportion of animals with B's. They were derived from single pair matings between individuals collected as fourth instars from Shakers Furze in Norfolk (grid reference 913955TL). 0B mating partners were predominantly reared from single pair matings between individuals collected as fourth instars from Winterton on the Norfolk coast (500200TG), although a few came from Shakers Furze single pair matings. Hoppers were hatched and reared to fourth instar (the subadult stage) under standard laboratory conditions using the methods described in Shaw (1981), based on those of Kelly-Stebbings and Hewitt (1972).

On reaching the fourth instar stage individuals were moved to their first adult environment. They were allocated to each of the three environments in turn so as to avoid any possible biases attributable to development rate. Similarly, the two progenies were allocated evenly among the environments as far as possible.

Females were allowed to mate with $\mathrm{OB}$ males as soon after their final moult as they were willing: usually $6-8$ days. The male was removed once the first pod was laid. After eight pods (25-35 eggs) had been laid, one-third of the females in each environment were moved to each of the other two environments and allowed to continue laying. This design allows the effects of environment after allowing for individual differences to be estimated, along with the effects of aging and individual differences themselves. Sibship differences or irreversible effects on the fourth instar and first adult environ- 
ment could be detected provided they were large relative to individual variation. A complete block of the design contains nine females: three replications, containing twenty-seven females, were originally set up.

By contrast, one-half the males allocated to each environment were mated immediately they became adult, while one-half were kept as virgins. Females were removed after 3 to 8 days, when the first pod was laid. After 10-12 days all the males were allowed to mate with new females for 2-3 days. (Thus, half the males mated more often than the other half.) After this, from each environment, one-third of the males were removed to each of the other two environments while one-third remained in place. After an acclimatisation period of 5-8 days, according to the availability of mature females, they were allowed to remate. After a further 10 days as many as possible were mated to a third or fourth female for 2-3 days. Finally, as many as remained alive were killed and the testis dissected out for determination of the karyotype.

This design allows the effects of age as such to be separated from the effects of number of matings made, since those males allowed four mates presumably mated more often than those allowed three. (In fact, it is likely that females will not often permit more than one mating in a short time interval: Shaw, 1981, ch. 11). As for the females, the labile effects of the environment and any individual differences can be detected, along with sufficiently large irreversible effects of the fourth instar and early adult environment and differences between the two sibships. Since each male produces several progenies it is possible if necessary to estimate differences between males separately from differences in the use of sperm by females. Lastly, since one-third of the males did not change environment after the initial allocation, the effects of changing environment per se can be distinguished from the effects of aging. However, the design has the drawback of being rather large, and it was only practicable to attempt a single replicate. As a result, premature deaths and incompatible matings lead to relatively low sensitivity to most of the effects mentioned.

In both the male and the female experiments the pods laid were kept for five to seven weeks at about $26^{\circ} \mathrm{C}$, then at $4^{\circ} \mathrm{C}$ for eight weeks or until we were ready to karyotype them, in order to break the embryo diapause. After three days at $26^{\circ} \mathrm{C}$ pods were dissected and embryos removed from the eggs into freshly prepared $3: 1$ ethanol:acetic acid fixative. Squash preparations in lactopropanoic orcein were used for karyotyping.

In all the crosses where the female was likely to contain a B, and in those where the male could not be karyotyped but was likely to contain a $\mathrm{B}$, the karyotype of the parents was deduced from the distribution of B's in the progeny (see Shaw, 1981, ch. 2).

\section{RESUltS}

Most of the analysis of the data gathered was carried out using the generalised linear model framework of Nelder and Wedderburn, 1972. A good introduction to this can be found in Baker and Nelder, 1975, but a very brief orientation will be given here so as to explain what follows to those unfamiliar with the techniques. For the purposes of statistical analysis, we assume that logit (probability of passing a B chromosome to an egg) is additively influenced by the various conditions manipulated during the 
experiment. The goodness of fit of a particular model to the data can be measured by the deviance. If there is no source of variation in the data beyond those assumed in the model, the deviance follows the $\chi^{2}$ distribution in large samples, so it can be compared with this as a test of the model. Similarly, the difference in deviance between two models can be used as a measure of whether one represents a better description of the data than another.

TABLE 1

Results of the female part of the experiment. " $H$ " stands for the hot environment, " $C$ " for the control, and " $L$ " for the low temperature environment. The two sibships used to provide B containing animals for the experiment were denoted "13" and " 1 ". Animals were named according to their first environment and the sequence in which they entered it

\begin{tabular}{ccccccc}
\hline Female & Sibship & B's & $\begin{array}{c}\text { First } \\
\text { environment }\end{array}$ & $\begin{array}{c}\text { 1B: Total } \\
\text { progeny }\end{array}$ & $\begin{array}{c}\text { Second } \\
\text { environment }\end{array}$ & $\begin{array}{c}\text { 1 B: Total } \\
\text { progeny }\end{array}$ \\
\hline H1 & 13 & 1 & H & $26: 27$ & L & $39: 40$ \\
H2 & 13 & 1 & H & $23: 26$ & H & $31: 36$ \\
H5 & 1 & 1 & H & $16: 22$ & H & $30: 36$ \\
H6 & 1 & 1 & H & $28: 29$ & C & $43: 45$ \\
H9 & 1 & 1 & H & $5: 7$ & C & $23: 40$ \\
L1 & 1 & 1 & L & $26: 36$ & L & $13: 23$ \\
L5 & 1 & 1 & L & $26: 28$ & L & $13: 21$ \\
L8 & 13 & 1 & L & $31: 35$ & L & $15: 16$ \\
L3 & 13 & 1 & L & $22: 24$ & H & $26: 35$ \\
L2 & 13 & 1 & L & $28: 38$ & C & $35: 47$ \\
L9 & 13 & 1 & L & $16: 16$ & C & $17: 20$ \\
C3 & 13 & 1 & C & $24: 27$ & - & $-18: 23$ \\
C7 & 1 & 1 & C & $19: 21$ & H & $28: 36$ \\
C4 & 1 & 1 & C & $27: 32$ & C & $10: 12$ \\
C6 & 13 & 1 & C & $9: 11$ & C & OB:1B:2B \\
& & & & 0B:1B:2B & & - \\
H3 & 13 & 2 & H & $1: 23: 13$ & - & - \\
H7 & 13 & 2 & H & $0: 8: 8$ & - & $0: 19: 11$ \\
H8 & 1 & 2 & H & $1: 8: 3$ & - & \\
H4 & 1 & 2 & H & $0: 9: 7$ & L & \\
& & & & OB:1B::2B:3B & & \\
C2 & 1 & 3 & C & $0: 18: 15: 5 *$ & & \\
\hline
\end{tabular}

* First and second environments both $C$; these totals are pooled.

\section{(i) Females}

The data collected are set out in table 1 . The experimental design finally achieved differs from that set out above for two reasons. First, some females proved infertile, did not carry a B, or died prematurely. Second, some possessed two $\mathrm{B}$ chromosomes, so that they cannot be analysed conveniently in the same statistical framework as the 1B's. An analysis of the 1B's in terms of the deviance of various models is set out in table 2.

There is a good deal of heterogeneity between the crosses (line 1), but most of this can be explained by variation between individuals (line 5). There is clealy no effect of environment (lines 7 and 8). Similarly, only a 
TABLE 2

Analysis of deviance of the female data, using 1 B' $^{\prime}$ only. The notation factor $1 /$ factor 2 implies "the effect of factor 1 after allowing for the effect of factor 2". The effect of a factor is the change in deviance produced by including it in the model used to fit the data

\begin{tabular}{|c|c|c|c|c|}
\hline Factor & $\begin{array}{l}\text { Residual } \\
\text { deviance }\end{array}$ & df & $\mathbf{P}$ & line \\
\hline \multicolumn{5}{|c|}{ a. Residual deviances after allowing for individual factors } \\
\hline None & $80 \cdot 2$ & 28 & 0.001 & 1 \\
\hline Environment & 78.9 & 26 & 0.001 & 2 \\
\hline Age & $73 \cdot 8$ & 27 & 0.001 & 3 \\
\hline Sibship & $73 \cdot 5$ & 27 & 0.001 & 4 \\
\hline Individual & $19 \cdot 4$ & 14 & $0 \cdot 15$ & 5 \\
\hline "High" & $53 \cdot 8$ & 27 & 0.001 & 6 \\
\hline \multicolumn{5}{|l|}{ b. Effects of individual factors } \\
\hline Environment & $1 \cdot 3$ & 2 & Not & 7 \\
\hline Environment/age and individual & 1.4 & 2 & significant & 8 \\
\hline Individual & $54 \cdot 1$ & 13 & 0.001 & 9 \\
\hline Age & $6 \cdot 4$ & 1 & 0.001 & 10 \\
\hline Age/individual & $5 \cdot 1$ & 1 & 0.01 & 11 \\
\hline "High" & $26 \cdot 4$ & 1 & - & 12 \\
\hline "High"/age & $25 \cdot 3$ & 1 & - & 13 \\
\hline
\end{tabular}

tiny fraction of the deviance can be explained by the first environment encountered (not shown). The factor "age"-that is" the contrast between the B-frequency in the first eight pods laid, and that in the second, regardless of environment-accounts for a substantial part of the variability even when differences between individuals are taken into account (lines 3,10 and 11). This suggests a real though slight decline in meiotic drive with age: a logit effect of about $0 \cdot 46$. The effect of sibship is as large as that of age, but less easy to interpret because it is confounded with the individual differences present in the data. The reduction in deviance due to fitting a sibship difference is only about one-tenth of that obtained by fitting individual differences. Inspection of table 1 shows that both the crosses with the least transmission of the B arise from sibship 1 and that they are very much lower than the average. Thus, the sibship effect arises not from a systematic difference between the sibships but from two aberrant individuals present in one of them. This point is emphasized by the "factor" "high" (lines 6, 12 and 13) which shows the improvement in fit effected by fitting a separate mean to these two individuals. Their presence probably indicates the segregation of a single genetic factor causing reduced meiotic transmission in this sibship (see Shaw, 1981 ch. 9 and 10).

To reiterate the important points, we find evidence of a slight decline in meiotic drive with age in remales, but no evidence of any environmental effects. Individual variability is large. As an example of the age-related change, a female with a transmission rate of 0.9 initially would have a transmission rate of 0.85 while laying her later pods.

\section{(ii) Males}

The data gathered in the male half of the experiment are set out in table 3. A larger proportion of the data comes from $2 B$ individuals, and there 


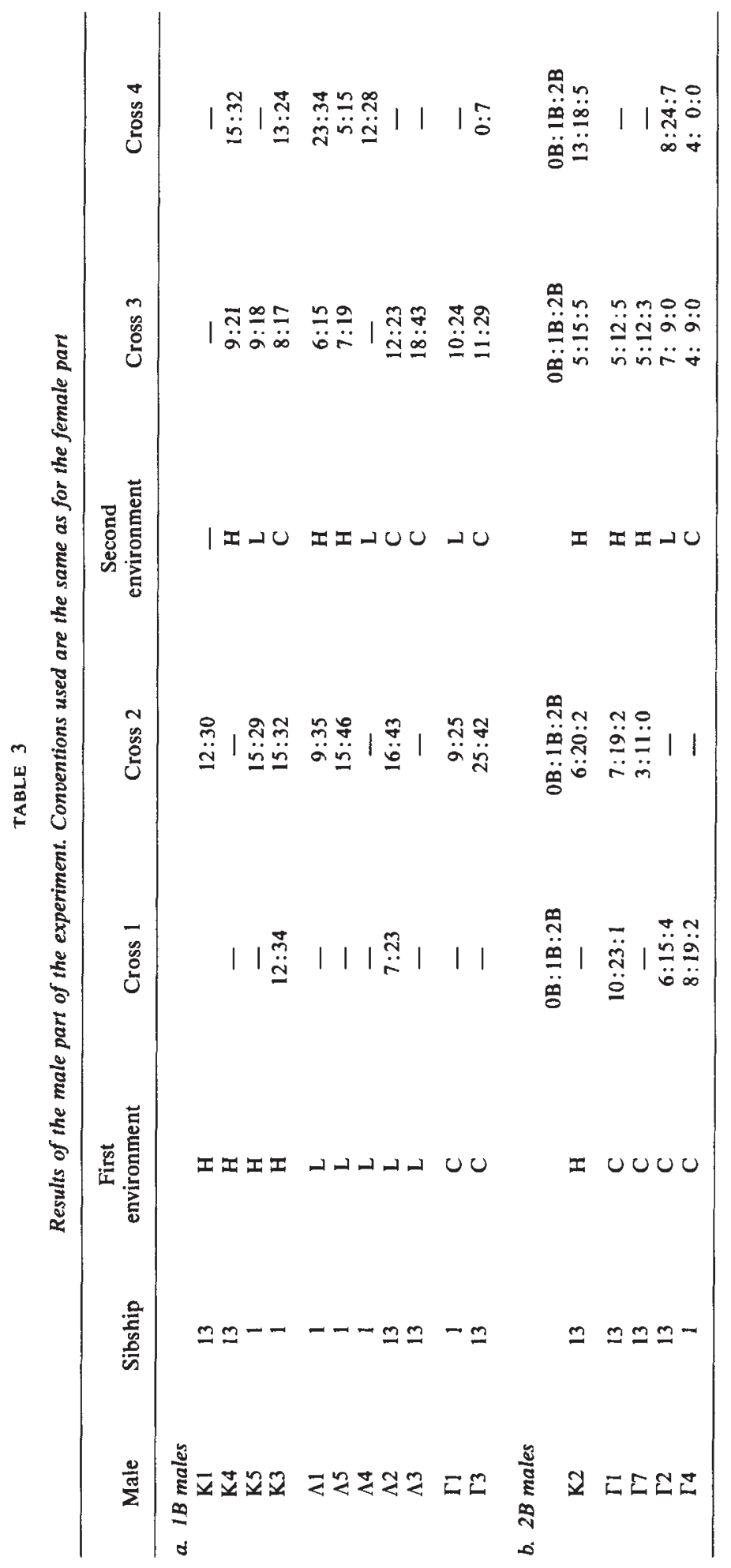


are substantial gaps in the design described in the previous section: the analysis is correspondingly more delicate. Table 4 sets out an analysis of deviance, like that described for the females, of the data arising from $1 \mathrm{~B}$ parents.

TABLE 4

Analysis of deviance of the male data, using $1 B^{\prime}$ 's only. The notation is as for the female analysis

\begin{tabular}{|c|c|c|c|c|}
\hline Factor & $\begin{array}{l}\text { Residual } \\
\text { deviance }\end{array}$ & $d f$ & $P$ & line \\
\hline \multicolumn{5}{|c|}{ a. Residual deviances after allowing for individual factors } \\
\hline None & $36 \cdot 0$ & 24 & 0.05 & 1 \\
\hline Environment & $26 \cdot 4$ & 22 & 0.24 & 2 \\
\hline Initial environment & 34.8 & 22 & 0.04 & 3 \\
\hline Sibship & $36 \cdot 0$ & 23 & 0.04 & 4 \\
\hline Age & $31 \cdot 7$ & 21 & 0.06 & 5 \\
\hline Individual & $30 \cdot 2$ & 14 & 0.008 & 6 \\
\hline \multicolumn{5}{|c|}{ b. Effects of individual factors } \\
\hline Environment & $9 \cdot 6$ & $2\}$ & Seet toxt & 7 \\
\hline Environment/individual & $14 \cdot 0$ & 2\} & setext & 8 \\
\hline Individual & 5.8 & 10 & Not & 9 \\
\hline Individual/environment & $10 \cdot 2$ & 10 & significant & 10 \\
\hline Age/environment & $3 \cdot 2$ & 3 & $\begin{array}{c}\text { Not } \\
\text { significant }\end{array}$ & 11 \\
\hline
\end{tabular}

The heterogeneity of the data is much less than for the females; the null hypothesis of homogeneity has a significance level of 0.05 almost exactly. Of the factors examined singly, only environment can economically explain any of the heterogeneity (lines 2 to 6 ). This remains true even after allowing for the possibility of intermale differences (line 8). After allowing for environment, none of the other factors improves the fit of the model significantly (lines 9 to 11 ).

The effect of environment has a significance level of between 0.025 and 0.05 when tested against the $F$ distribution without allowing for the effect of possibly different individual means. (See Baker and Nelder, 1975 for a discussion of the validity of this:' for data like these it is certainly no less valid than a conventional $F$ test on the results of an analysis of variance.) Alternatively, testing the effect against $\chi^{2}$ after allowing for individual differences one obtains a significance level of 0.001 . The corresponding mean transmission levels in each environment are 0.46 in the hot, 0.48 in the control and 0.35 in the low.

Since the means in hot and control environments are so similar, it seems reasonable to regard them as variants of a single temperature regime for the purposes of comparison with the low environment. Table 5 sets out the means for each individual in the experiment. It is clear that three of the six males swapped between hot or control and low environments showed large differences, while three showed almost no change. In fact, for this group of six males the total 2G (Sokal and Rohlf, 1969) for differences between the two environments is 18.52 , of which $\Lambda 1, \Lambda 2$, and $\Gamma 3$ contribute $18 \cdot 3$. It seems hard to avoid the conclusion that there is a real effect of environment on some males, but not on others. 
TABLE 5

Transmission rates of males used in the experiment in the hot/control environment or in the cold one. Transmission rates of $2 B$ 's have been calculated as (total $B$ 's observed $) /($ total egg observed), so that the mendelian expectation would be 1.0

\begin{tabular}{ccc}
\hline Individual & $\begin{array}{c}\text { Transmission } \\
\text { in: Hot } \\
\text { or control }\end{array}$ & Cold \\
\hline K1 & 0.40 & \\
K3 & 0.45 & \\
K4 & 0.45 & \\
K5 & 0.52 & 0.50 \\
$\Lambda 1$ & 0.59 & 0.26 \\
$\Lambda 2$ & 0.52 & 0.35 \\
$\Lambda 5$ & 0.35 & 0.33 \\
$\Gamma 1$ & 0.39 & \\
$\Gamma 3$ & 0.59 & 0.31 \\
K2 & 0.87 & \\
T1 & 0.83 & 0.85 \\
$\Gamma 2$ & 0.92 & \\
$\Gamma 4$ & 0.70 & 0.43 \\
$\Gamma 7$ & 0.85 & \\
$\Lambda 4$ & & \\
\hline
\end{tabular}

\section{Discussion}

The effect of age in reducing female transmission, although unambiguous, is small and unlikely to have much influence on the geographic distribution of B chromosomes. This is probably true whether the effect is a true one of age, or a consequence of the total number of eggs laid. In the first case, Robinson (1973) suggests that the average lifespan of a female adult is of the order of three weeks-much shorter than the duration of this experiment-while in the second, the same author estimates that an average female lays only three to five pods in her lifetime. The effect needs to be borne in mind during the planning and analysis of laboratory experiments but the small correction required will usually be negligible relative to binomial sampling error in the $\mathrm{B}$ frequencies among individual progenies.

Otherwise, the experiment makes clear that effects of temperature over the range studied during subadult and adult phases are likely to be small. Since equilibrium B frequency is smoothly related to the parameters controlling it (Shaw, 1984), this means that temperature is unlikely to act markedly on equilibrium $B$ frequency through the female transmission rate.

The effect of temperature on average male transmission, on the other hand, is striking and could be a perfectly plausible explanation of the variation in B frequency observed by Hewitt and Ruscoe, or of the overall distribution pattern of the B chromosome in the U.K. However, interpretation needs to be cautious. Grasshoppers in the wild undoubtedly bask and shade themselves in order to regulate their temperature, as mentioned before, while the animals in this experiment were deliberately prevented from doing so. Thus the temperature regime recorded in the wild by passive meteorological instruments may be quite different from that perceived by the grasshop- 
pers. Furthermore, the present experiment gives no indication of the particular features of the temperature regime responsible for the differences seen, although the similarity of the hot and control environments suggests that the response may take place at particular thresholds.

The differences in the response shown by different males are notable. Since all the young were brought up in environments intended to be as similar as possible and those showing or not showing a particular response to temperature were not reared together, it is possible that the response differences are genetic. The animals which responded to temperature came from both progenies, but this does not contradict the hypothesis. If it were to be correct, the genetic factor or factors responsible are in effect temperature sensitive modifiers of meiotic drive. In a population experiencing a suitably cool climate the theory set out in Shaw (1984) should apply. This suggests that the modifiers would increase in frequency, reducing the average net meiotic drive and hence the equilibrium B frequency, until their pleiotropic effects were comparable in selective importance with their effects on B chromosome transmission frequency. In turn, of course, B chromosomes insensitive to the reduction in transmission will tend to increase in frequency and a constantly evolving frequency-dependent balance will result, provided suitable genetic variation exists.

Whatever the case, our data suggest that reduction in net meiotic drive with temperature is a possible cause of variation in observed B frequency and experiments on the topic might be worthwhile in the field. A possible method might be to pick two sites with $B$ frequencies known to be stable and different and then to release marked virgin females into the population. These could be followed until they mated, the mating pairs captured and the female allowed to lay in the laboratory. In this way, substantial clutches of eggs from individual matings might be obtained and the problems introduced by multiple matings avoided. Laboratory matings of males from both populations and temperature recordings would complete the picture. The experiments would be time-consuming but could be very rewarding.

Acknowledgements. During this work MWS was supported initially by a Natural Environment Research Council postgraduate studentship and latterly by a similar award from the University of East Anglia. He is grateful to both bodies. We would also like to thank Range Control, Stanford Battle Training Area, for permission to collect at Shakers Furze.

\section{REFERENCES}

BAKER, R. J. AND NELDER, J. A. 1978. The GLIM system, release 3: manual. Royal Statistical Society, London.

GREGG, P. 1981. The adaptation of a locust to its physical environment. PhD thesis, Australian Nat. Univ.

HEWITT, G. M. 1973. Variable transmission rates of a B chromosome in Myrmeleotettix maculatus (Thunb.) (Acrididae: Orthoptera). Chromosoma, 40, 83-106.

HEWITT. G. M. AND BROWN, F. M. 1970. The B-chromosome system of Myrmeleotettix maculatus. V. A steep cline in East Anglia. Heredity, 25, 363-371.

HEWITT, G. M. AND JOHN, B. 1967. The B-chromosome system of Myrmeleotettix maculatus. III. The statistics. Chromosoma, 21, 140-162.

HEWITT, G. M. AND RUSCOE, C. N. E. 1971. Changes in microclimate correlated with a cline for B-chromosomes in the grasshopper Myrmeleotettix maculatus. J. Anim. Ecol., 40, 753-765.

KELLY-STEBBINGS, A. AND HEWITT, G. M. 1972. The laboratory breeding of British gomphocerine grasshoppers (Acrididae: Orthoptera). Acrida, 1, 233-245. 
NElder, J. A. AND WedDerburn, R. W. 1972. Generalised linear models. J. Roy. Stat. Soc. $A, 125,370-384$.

ROBINSON, P. M. 1973. The ecological genetics of the polymorphism for B-chromosome in the grasshopper Myrmeleotettix maculatus. (Thunb.) (Acrididae: Orthoptera). Ph.D thesis, Univ. of East Anglia.

SHAW, M. W. 1981. A B chromosome cline in the mottled grasshopper. Ph.D. thesis, Univ. of East Anglia.

SHAW, M. W. 1983. Rapid movement of a B-chromosome frequency cline in Myrmeleotettix maculatus (Orthoptera: Acrididae). Heredity, 50, 1-14.

SHAW, M. W. (1984). The population genetics of the B chromosome polymorphism of Myrmeleotettix maculatus (Orthoptera: Acrididae). Biol. J. Linn. Soc., 23 (in press).

SOKAL, R. R. AND ROHLF, F. J. 1969. Biometry. Freeman: San Francisco. 